\title{
Surface Degradation Analysis of Commercial Nickel-rich Oxide Cathode Materials by Multiple Electron Microscopy Technologies
}

Jiangtao Zhu, Xiuhong Han, Jane Sun, Shaojie Wang, Tina Hsu, Yanxiao Ma, Zhihuan Wang, Christopher Kurtz, Xinyu Zhao, Charlene Sun, Cynthia Wang and Jiajie Qian

Eurofins Nanolab Technologies, United States

With the increasing demand for energy storage, it is critical to understand the microstructure and composition of lithium-ion materials at high spatial resolution. Nickel rich cathode materials are promising, especially for electric vehicle (EV) batteries due to their high capacity and low cost. In Ni rich cathodes, $\mathrm{Mn}$ or $\mathrm{Al}$ are usually added to stabilize the structure. Once the Ni content increases, the energy density will increase, however, Ni rich cathodes suffers performance degradation because of their structure, composition and phase change caused by $\mathrm{Ni} / \mathrm{Li}$ cationic mixing, oxygen evolution, irreversible layered-spinel-rock salt phase transition, etc [1].

In order to fully understand the structure, composition and phase of the cathode materials, multiple techniques are necessary. Aberration-corrected Scanning Transmission Electron Microscopy (AC-STEM) is a great technique to understand the local structure change at atomic level [2]. Energy Dispersive Spectroscopy (EDS) and Electron Energy Loss Spectroscopy (EELS) which are coupled with STEM, can provide composition analysis including distribution of each element. EELS is able to detect $\mathrm{Li}$ and understand Li distribution [3]. Precession Electron Diffraction (PED), can directly provide phase maps on the degraded surface of the grains [4]. Eurofins Nanolab Technologies, Inc (Milpitas, CA, USA) has a large variety of electron microscopy techniques such as mechanical polishing, scanning electron microscopy, focused ion beam (FIB), AC-STEM, EDS/EELS and PED.

A used commercial EV battery was ordered and discharged before being cut to observe the cross section. The battery has a jelly-roll structure with alternating layers of anode and cathode (Figure 1a,b). A TEM lamella was extracted from the cathode film using the FIB lift-out technique on a dual beam FIB/SEM system. A probe-corrected Titan equipped with Gatan Enfinium EELS was operated at 200kV for STEM imaging and EDS/EELS analysis. As shown in Figure 1c, secondary grains consisted of agglomerated primary grains with a microcrack inside. There are also some loose primary particles. A shell layer was observed on a grain which is close to a microcrack (Figure. 1d). This shell is not visible in the surface of the same grain away from the crack. The core of the grain has a welldefined layered structure without any sign of Ni/Li mixing (Figure 1f); however, Ni atoms entered the Li positions at the shell layer. Inside the shell, the very top surface of the shell has a rock-salt structure, beneath that is the mixed structure of both rock-salt and layer structure (Figure 1e). These shell layers will give ionic and electrical resistance of the battery and cause degradation.

The cathode material is identified to be lithium nickel cobalt aluminum oxides (NCA) by EELS/EDS analysis. The elemental maps show the absence of $\mathrm{Li}$ at the shell layer (Figure 2), while the $\mathrm{Ni}, \mathrm{Co}$ and $\mathrm{Al}$ were distributed across the whole grain. At the very top surface $(\sim 2 \mathrm{~nm})$ of the shell layer, the Ni L edge shifts to lower energy loss and O$\mathrm{K}$ pre-edge intensity drops, which indicates the formation of NiO. This is also consistent with the STEM images that indicate the presence of rock-salt structure. The PED phase maps confirm the presence of $\mathrm{NiO}$ and can provide the phase map at large scale ( um). The degradation happened at the surface of the secondary grains and the primary grains which were near the crack. The grain orientation maps obtained by PED revealed the grains have a random orientation without texture structure. In summary, AC-STEM, EDS/EELS and PED together can provide structure, composition and phase analysis to further understand the degradation of the cathode materials. 
(a)

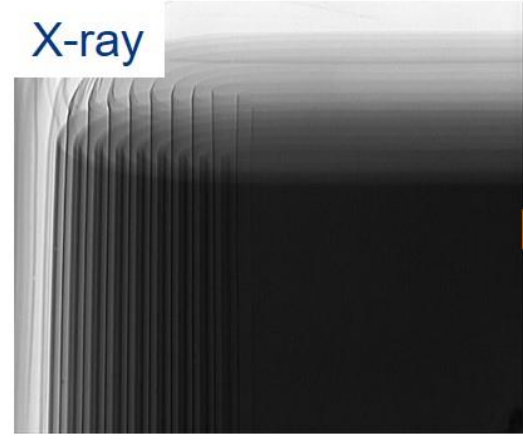

(f)

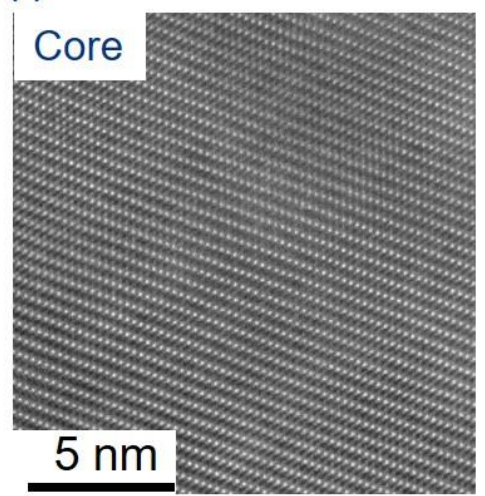

(b)

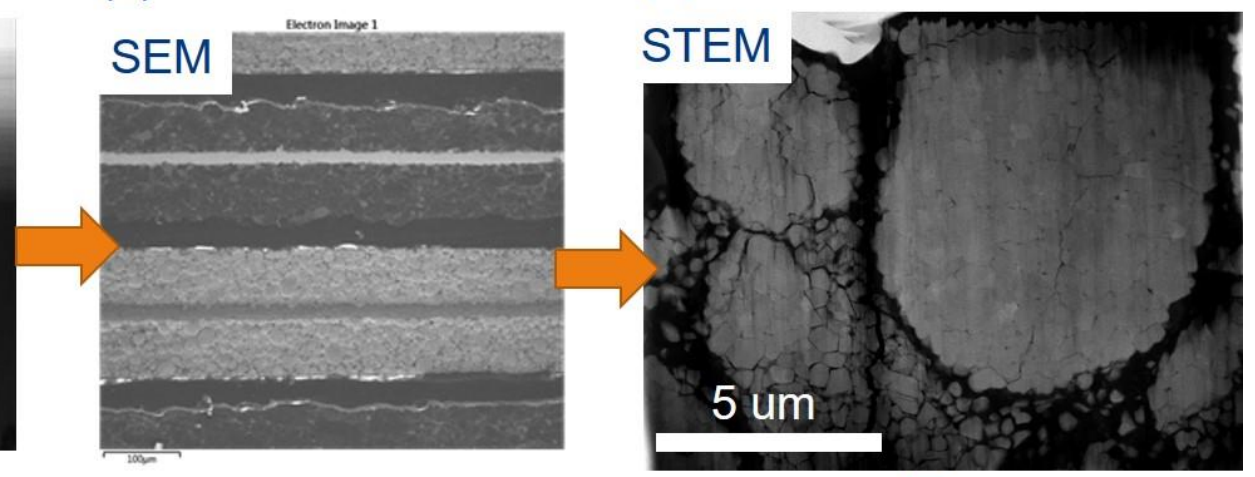

(e)

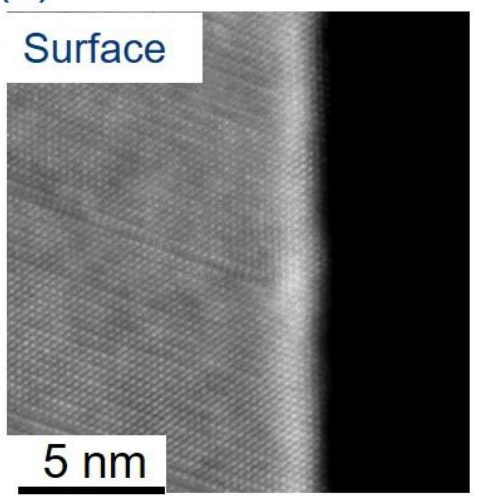

(c)

(d)

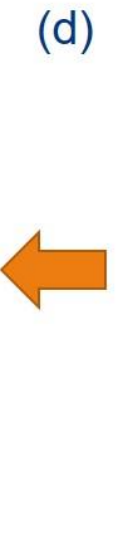

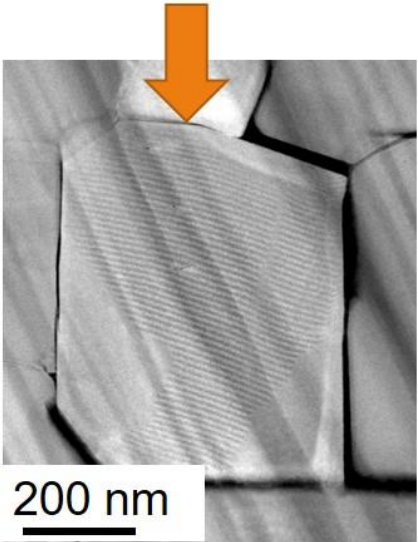

Figure 1. Figure 1. Jelly-roll like structure of an EV battery at different scales (a) x-ray image, (b) SEM cross section image. STEM images of (c) cathode secondary grains and (d) a grain near a microcrack has a shell layer at its right. High resolution STEM images show the rock-salt (e) and layered (f) structure at the surface and the core of the grain in (d).

(a)

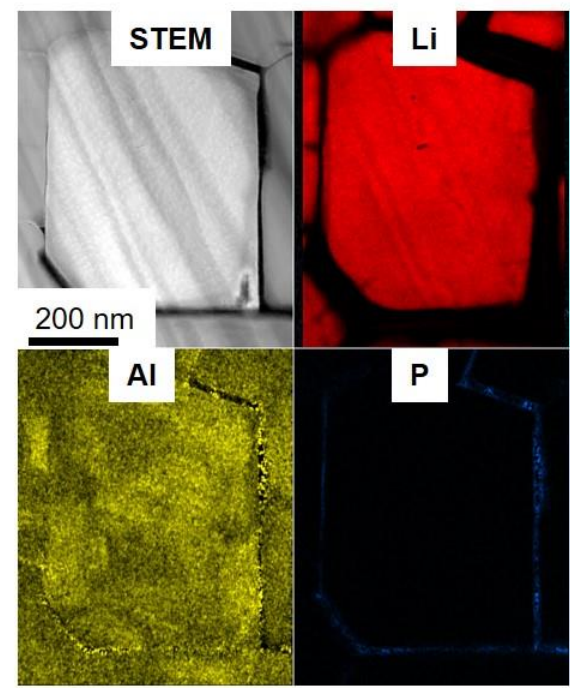

(b) NCA $\mathrm{NiO}$

(c)
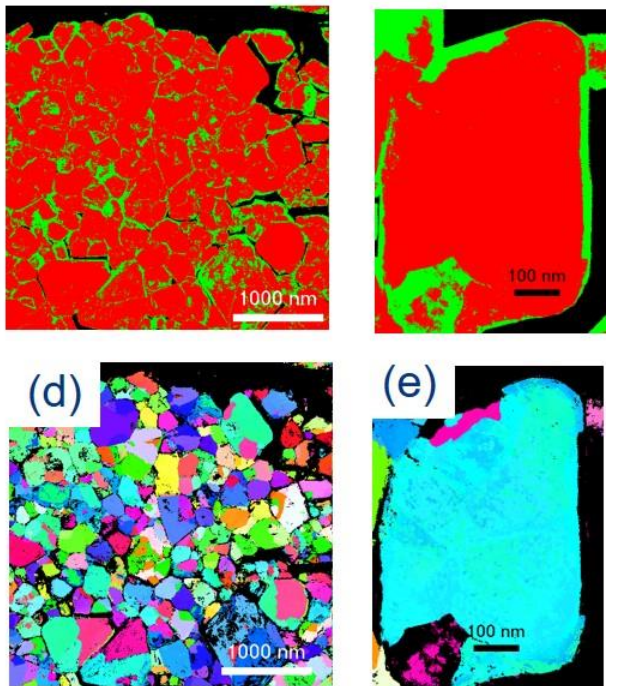

Figure 2. Figure 2. (a) STEM/EELS/EDS maps show the absence of Li at the shell surface and homogeneous distribution of $\mathrm{O}, \mathrm{Ni}, \mathrm{Co} . \mathrm{Al}$ is not uniform across the grain. $(\mathrm{b}, \mathrm{c})$ phase maps of NCA and $\mathrm{NiO}$ and $(\mathrm{d}, \mathrm{e})$ grain orientation maps of NCA by PED 


\section{References}

[1] S. Zhang, Energy Storage Mater, 24 (2020), p. 247.

[2] A. Boulineau, Nano Lett, 13 (2013), p.3857.

[3] Y. Nomura, Nature Comm, 11(2020), p.3622.

[4] G. Brunetti, Chem Mater, 23(2011), p.4515. 